\section{Balloon pump-induced pulsatile perfusion during cardiopulmonary bypass does not improve brain oxygenation}

\section{To the Editor:}

We read with interest the article by Kawahara and associates regarding the effects of pulsatile versus nonpulsatile perfusion on internal jugular venous oxygen saturation and regional cerebral venous oxygen saturation during normothermic cardiopulmonary bypass (CPB) in 22 patients. ${ }^{1}$ They have concluded that when compared with nonpulsatile flow, pulsatile perfusion generated by an intra-aortic balloon pump had no beneficial effect on cerebral protection.

The authors stated that although many methods have been used to generate pulse pressure during $\mathrm{CPB}$, no general definition and no criteria have been reported for pulsatile perfusion. In contrast, the literature reports that several investigators have attempted to establish a common criterion for pulsatile and nonpulsatile flow. ${ }^{2-6}$ Shepard, Simpson, and Sharp ${ }^{2}$ suggested that the energy equivalent pressure (EEP) formula may be used to quantify pulsatile and nonpulsatile waveforms. The generation of the pulsatile flow depends on the energy gradient rather than the pressure gradient. EEP contains both pump flow and arterial pressure waveforms. The following formula is used to define the EEP:

$$
\mathrm{EEP}=\left(\int \mathrm{fpdt}\right) /\left(\int \mathrm{fdt}\right)
$$

where $p$ is the arterial pressure, $f$ is the pump flow, and dt is the change in time at the end of flow and pressure cycles. The units for EEP are millimeters of mercury. EEP is the ratio of the area under the hemodynamic power curve ( $($ fpdt) and the flow curve ( ffdt) at the end of flow and pressure cycles.

Recently, we have quantified pulsatile and nonpulsatile waveforms in terms of EEP. ${ }^{7}$ With an identical pump flow rate and mean arterial pressure, the pulsatile roller pump (Stöckert SIII, Munich, Germany) generated significantly higher EEP than did conventional nonpulsatile perfusion. In addition, we have shown that this increase in EEP maintained higher regional and global cerebral, renal, and myocardial blood flow in a neonatal piglet model. ${ }^{8}$ In a separate study with a different pulsatile roller pump (Jostra HL-20, Jostra USA, Austin, Tex), we have shown that regional cerebral venous oxygen saturation increased during normothermic and hypothermic $\mathrm{CPB}$ in a neonatal piglet model. ${ }^{9}$ In this particular study, EEP was significantly higher than mean arterial pressure.

Our experience leads us to the conclusion that EEP is the most complete formula to quantify pulsatile and nonpulsatile waveforms for direct comparisons.

Akif Ündar, PhD ${ }^{a, b, c}$ Congenital Heart Surgery Service ${ }^{a}$ Texas Children's Hospital 6621 Fannin St, MC 1-2285

Houston, TX 77030-2399 Charles D. Fraser, $J r, M D^{a, c, d}$ Cullen Cardiovascular Surgical Research Laboratories ${ }^{b}$ Texas Heart Institute Houston, TX 77225

Departments of Surgeryc and Pediatrics ${ }^{d}$ Baylor College of Medicine Houston, TX 77030

\section{REFERENCES}

1. Kawahara F, Kadoi Y, Saito S, Yoshikawa D, Goto F, Fujita N. Balloon pump-induced pulsatile perfusion during cardiopulmonary bypass does not improve brain oxygenation. J Thorac Cardiovasc Surg 1999;118:361-6.

2. Shepard RB, Simpson DC, Sharp JF. Energy equivalent pressure Arch Surg 1966;93:730-40.

3. Wright G, Furness A. What is pulsatile flow? Ann Thorac Surg 1985;39:401-2.

4. Wright G. Hemodynamic analysis could resolve the pulsatile blood flow controversy. Ann Thorac Surg 1994;58:1199-204.

5. Ündar A. Design and performance of physiologic pulsatile flow cardiopulmonary bypass systems for neonates and infants. $\mathrm{PhD}$ dissertation, The University of Texas at Austin, May 1996.

6. Ündar A, Frazier OH, Fraser CD. Defining pulsatile perfusion: quantification in terms of energy equivalent pressure. Artif Organs 1999;23:712-16.

7. Ündar A, Masai T, Frazier OH, Fraser CD. Pulsatile and non-pulsatile flows can be quantified in terms of energy equivalent pressure during cardiopulmonary bypass for direct comparisons. ASAIO J. In press.

8. Ündar A, Masai T, Yang SQ, Goddard-Finegold J, Frazier OH, Fraser CD. Effects of perfusion mode on regional and global organ blood flow in a neonatal piglet model. Ann Thorac Surg 1999;68:1336-43.

9. Ündar A, Eichstaedt HC, Frazier OH, Fraser CD. Monitoring regional cerebral oxygen saturation using near-infrared spectroscopy during pulsatile hypothermic cardiopulmonary bypass in a neonatal piglet model. ASAIO J. In press.

$12 / 8 / 103297$

\section{Reply to the Editor:}

We appreciate the interest and comments of Drs Ündar and Fraser. Several investigators ${ }^{1}$ have tried to establish common criteria for pulsatile flow, but it is not clear which type of pulsatile waveform has positive effects on cerebral circulation and improves outcome of patients. ${ }^{2}$ As stated by Ündar and associates, ${ }^{3}$ it is impossible to compare the results of different investigations in which different types of pulsatility were used.

We can see that the concept of energy equivalent pressure introduced by Shepard, Simpson, and Sharp ${ }^{4}$ is useful for understanding the quality of pulsatile perfusion. Wright ${ }^{5}$ believes that hemodynamic considerations were fundamental to resolve the controversy of the pulsatility waveform. We hope that further clinical investigations will determine the best pulsatile form and will be supported by logical theories, such as energy equivalent pressure.

Yuji Kadoi, $M D$

Shigeru Saito, MD

Department of Anesthesiology and Reanimatology Gunma University, School of Medicine 3-39-22, Showa-machi Maebashi, Gunma 371-8511, Japan

\section{REFERENCES}

1. Lodge AJ, Under K, Daggett CW, Runge TM, Calhoon JH, Ungerleider RM. Regional blood flow during pulsatile cardiopulmonary bypass and after circulatory arrest in an infant model. Ann Thorac Surg 1997;63:1243-50. 